\title{
Development of MK-8353, an orally administered ERK1/2 inhibitor, in patients with advanced solid tumors
}

Stergios J. Moschos, ${ }^{1}$ Ryan J. Sullivan, ${ }^{2}$ Wen-Jen Hwu, ${ }^{3}$ Ramesh K. Ramanathan, ${ }^{4}$ Alex A. Adjei, ${ }^{5}$ Peter C. Fong, ${ }^{6}$ Ronnie Shapira-Frommer, ${ }^{7}$ Hussein A. Tawbi, ${ }^{8}$ Joseph Rubino, ${ }^{9}$ Thomas S. Rush III, ${ }^{9}$ Da Zhang, ${ }^{9}$ Nathan R. Miselis, ${ }^{9}$ Ahmed A. Samatar, ${ }^{9}$ Patrick Chun, ${ }^{9}$ Eric H. Rubin, ${ }^{9}$ James Schiller, ${ }^{9}$ Brian J. Long, ${ }^{9}$ Priya Dayananth, ${ }^{9}$ Donna Carr, ${ }^{9}$ Paul Kirschmeier, ${ }^{9}$ W. Robert Bishop, ${ }^{9}$ Yongqi Deng, ${ }^{9}$ Alan Cooper, ${ }^{9}$ Gerald W. Shipps, ${ }^{9}$ Blanca Homet Moreno, ${ }^{10}$ Lidia Robert, ${ }^{10}$ Antoni Ribas, ${ }^{10}$ and Keith T. Flaherty ${ }^{2}$

'Lineberger Comprehensive Cancer Center, University of North Carolina at Chapel Hill, Chapel Hill, North Carolina, USA. ${ }^{2}$ Massachusetts General Hospital Cancer Center, Boston, Massachusetts, USA. ${ }^{3}$ Department of Melanoma Medical Oncology, The University of Texas MD Anderson Cancer Center, Houston, Texas, USA. ${ }^{4}$ Translational Genomics Research Institute, Phoenix, Arizona, USA; Virginia G. Piper Cancer Center, Scottsdale, Arizona, USA. ${ }^{5}$ Department of Medicine, Roswell Park Cancer Institute, Buffalo, New York, USA. ${ }^{6}$ The University of Auckland and Auckland City Hospital, Auckland, New Zealand. 'Department of International Medicine, Chaim Sheba Medical Center, Tel-HaShomer, Israel. ${ }^{8}$ University of Pittsburgh School of Medicine, University of Pittsburgh Cancer Institute, Pittsburgh, Pennsylvania, USA. ${ }^{9}$ Merck \& Co. Inc., Kenilworth, New Jersey, USA. ${ }^{10}$ Jonsson Comprehensive Cancer Center at UCLA, University of California Los Angeles, Los Angeles, California, USA.

Role of funding source: Merck Sharp \& Dohme Corp., a subsidiary of Merck \& Co. Inc., was responsible for preclinical development of MK-8353 as well as design and conduct of healthy volunteer (P07652) and advanced solid tumor phase I (MK-8353-001) studies and assisted in manuscript preparation and submission. $\mathrm{NIH}$ grants (PO1 CA168585 and R35 CA197633) provided funds for tumor biopsy collection of fresh tumor tissue as part of the MK-8353-001 study and establishment of patient-derived M428 and M435 melanoma cell lines in preparation for cell proliferation studies using ERK and MEK inhibitors.

Submitted: December 21, 2016 Accepted: December 28, 2017 Published: February 8, 2018

Reference information:

JCI Insight. 2018;3(3):e92352. https:// doi.org/10.1172/jii.insight.92352.
BACKGROUND. Constitutive activation of ERK1/2 occurs in various cancers, and its reactivation is a well-described resistance mechanism to MAPK inhibitors. ERK inhibitors may overcome the limitations of MAPK inhibitor blockade. The dual mechanism inhibitor SCH772984 has shown promising preclinical activity across various $B R A F^{v 600} / R A S$-mutant cancer cell lines and human cancer xenografts.

METHODS. We have developed an orally bioavailable ERK inhibitor, MK-8353; conducted preclinical studies to demonstrate activity, pharmacodynamic endpoints, dosing, and schedule; completed a study in healthy volunteers (P07652); and subsequently performed a phase I clinical trial in patients with advanced solid tumors (MK-8353-001). In the P07652 study, MK-8353 was administered as a single dose in 10- to 400-mg dose cohorts, whereas in the MK-8353-001 study, MK-8353 was administered in 100- to $800-\mathrm{mg}$ dose cohorts orally twice daily. Safety, tolerability, pharmacokinetics, pharmacodynamics, and antitumor activity were analyzed.

RESULTS. MK-8353 exhibited comparable potency with SCH772984 across various preclinical cancer models. Forty-eight patients were enrolled in the P07652 study, and twenty-six patients were enrolled in the MK-8353-001 study. Adverse events included diarrhea (44\%), fatigue (40\%), nausea (32\%), and rash ( $28 \%$ ). Dose-limiting toxicity was observed in the $400-\mathrm{mg}$ and $800-\mathrm{mg}$ dose cohorts. Sufficient exposure to MK- 8353 was noted that correlated with biological activity in preclinical data. Three of fifteen patients evaluable for treatment response in the MK-8353-001 study had partial response, all with $B R A F^{1600}$-mutant melanomas.

CONCLUSION. MK-8353 was well tolerated up to $\mathbf{4 0 0}$ mg twice daily and exhibited antitumor activity in patients with $B R A F^{v 600}$-mutant melanoma. However, antitumor activity was not particularly correlated with pharmacodynamic parameters.

TRIAL REGISTRATION. ClinicalTrials.gov NCT01358331.

FUNDING. Merck Sharp \& Dohme Corp., a subsidiary of Merck \& Co. Inc., and NIH (P01 CA168585 and R35 CA197633). 


\section{Introduction}

ERK1/2 are members of the MAPK family of signaling proteins, a superfamily of cytoplasmic serine/threonine kinases that normally transmit signals from various extracellular stimuli to intracellular targets and regulate a number of cellular processes, including proliferation, adhesion, migration, survival, differentiation, and metabolism $(1,2)$. Activating mutations in upstream effectors that result in constitutive MAPK activation are present in almost $35 \%$ of all cancers (3). Reactivation of the ERK1/2 phosphorylation and signaling has been frequently associated with secondary resistance to BRAF and MEK inhibitors $(4,5)$, highlighting the importance of exploring targeted inhibition of more downstream components of the MAPK signaling pathway. In fact, in $B R A F^{V 600}$-mutant melanoma cell lines that were established from patients who developed resistance to MAPK pathway inhibitors, pharmacologic inhibition of ERK1/2 was more potent than MEK inhibition in suppressing MAPK activity and cell proliferation and enhancing cell death (6).

While the development of small-molecule inhibitors of RAS currently remains pharmacologically challenging (reviewed in ref. 7), a number of ERK inhibitors (ERKi) have been successfully developed and are currently in early clinical development $(8,9)$. These compounds have been shown to either disrupt ERK dimerization (10) or to bind to the active conformation, inhibit intrinsic kinase activity, and, therefore, prevent phosphorylation of downstream kinases ("single mechanism," ATP-competitive, type I) (11-13). SCH772984 is a unique ERK1/2 inhibitor, because it does not only bind to and inhibit ERK1/2-intrinsic kinase activity, but it also induces a conformational change upon binding to ERK1/2 that prevents ERK1/2 phosphorylation by upstream kinases ("dual mechanism") $(11,14,15)$. More specifically, SCH772984 potently and selectively inhibits ERK1 and ERK2 activity, with $\mathrm{IC}_{50}$ values of 4 and $1 \mathrm{nM}$, respectively. Treatment of $B R A F /$ $R A S$-mutant melanoma and colon cancer cell lines with SCH772984 led to sustained inhibition of the downstream effectors of ERK1/2 signaling, transient inhibition of MEK-mediated ERK phosphorylation (pERK), decreased cell proliferation, and increased apoptosis. In addition, SCH772984 suppressed proliferation of melanoma and colorectal cancer cell lines that had developed secondary resistance to BRAF/MEK inhibitors $(16,17)$. Finally, concurrent administration of vemurafenib and SCH772984 in various melanoma cell lines was significantly more potent than that with either agent alone (17).

Based on these results, we developed an orally bioavailable dual mechanism ERKi, MK-8353 (formerly SCH900353), with improved pharmacokinetic properties compared with the preclinical tool compound SCH772984. Preclinically, MK-8353 showed similar selectivity and potency to SCH772984. Antitumor activity was comparable in various preclinical models. Following an early study of MK-8353 administered as a single dose in healthy human subjects (P07652), we performed a phase I trial of MK-8353 in patients with advanced solid tumors (MK-8353-001). The first objective in the MK-8353-001 clinical trial was to define the dose-limiting toxicity (DLT) and maximum tolerated dose (MTD) of MK-8353 and to determine the recommended phase 2 dose (RP2D). The second objective was to evaluate the antitumor efficacy of MK-8353 in patients with metastatic melanoma and colorectal cancer bearing BRAF or RAS mutation(s).

\section{Results}

$M K-8353$ is a highly potent, orally bioavailable, dual-specificity ERK1/2 inhibitor. $\mathrm{MK}-8353\left[\mathrm{HCl}\left(\mathrm{C}_{37} \mathrm{H}_{42} \mathrm{CI}-\right.\right.$ $\mathrm{N}_{9} \mathrm{O}_{3} \mathrm{~S}$ ), molecular weight, 728.31, Figure 1A] is an orally bioavailable, selective, and potent ERKi that inhibits activated ERK1 and ERK2 in vitro, with $\mathrm{IC}_{50}$ values of $23.0 \mathrm{nM}$ and $8.8 \mathrm{nM}$, respectively (IMAP kinase assay), and nonactivated ERK2, with an $\mathrm{IC}_{50}$ of $0.5 \mathrm{nM}$ (MEK1-ERK2-coupled assay). MK-8353 demonstrates kinase selectivity over a 227-human kinase panel; no additional kinase in the panel was inhibited by more than $35 \%$ at the $0.1 \mu \mathrm{M}$ concentration, and only 3 kinases (CLK2, FLT4, and Aurora B) were inhibited $>50 \%$ at the $1.0 \mu \mathrm{M}$ concentration (data not shown). Similar to SCH772984, MK-8353 caused a dose-proportional decrease in the phosphorylated-activated forms of ERK1 (pERK1), ERK2 (pERK2), and ribosomal S6 kinase (pRSK) proteins, with complete suppression of pERK1 and pERK2 observed at $30 \mathrm{nM}$ MK-8353 in A2058 cells (Figure 1B). Additionally, MK-8353 inhibited the in vitro proliferation of a panel of $B R A F^{V 600}$-mutant and $R A S$-mutant cancer cell lines (Supplemental Table 1; supplemental material available online with this article; https://doi.org/10.1172/jci.insight.92352DS1).

The in vivo antitumor activity of MK-8353 was evaluated in a panel of human xenograft models, including the BRAFV600 mutant Colo-205 colon cancer model and the BRAFV600 mutant SK-MEL-28 melanoma model (Supplemental Table 2). MK-8353 inhibited the growth of both models in a dose-dependent manner at dose levels of 30-60 mpk, by oral gavage, twice daily. The $30 \mathrm{mpk}$ dose significantly inhibited the growth of both models, while the $60 \mathrm{mpk}$ dose led to tumor regressions (Figure 2, A and B). 
A

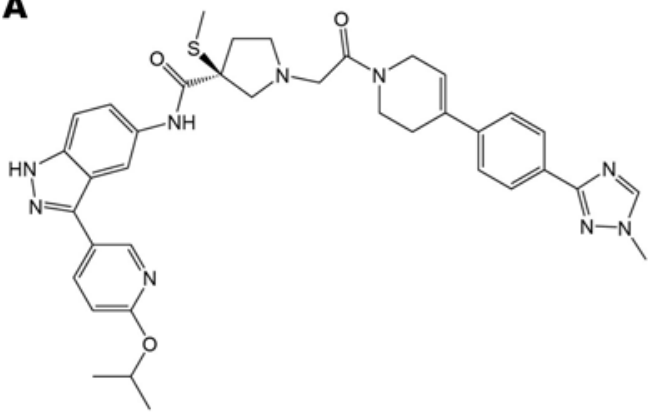

B

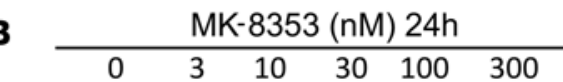

pERK

tERK

pRSK

tRSK

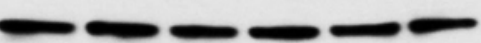

C

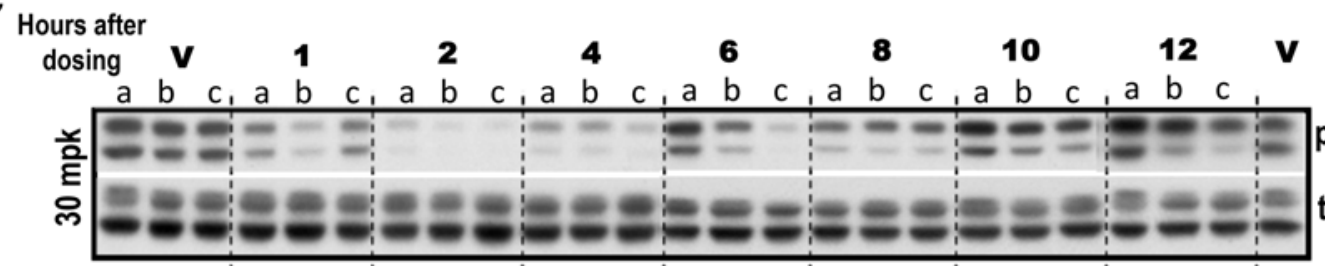

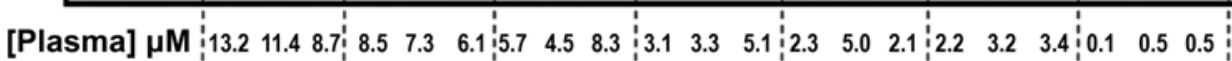

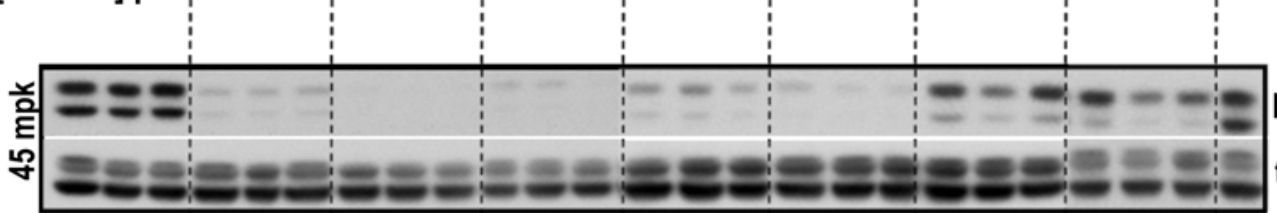

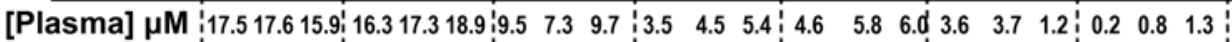

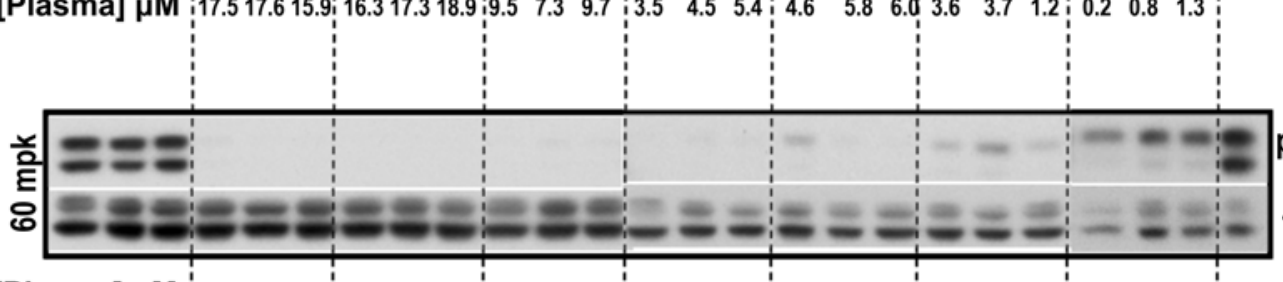

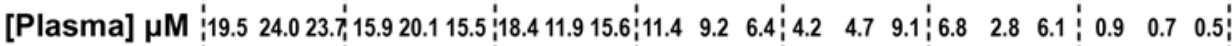

D

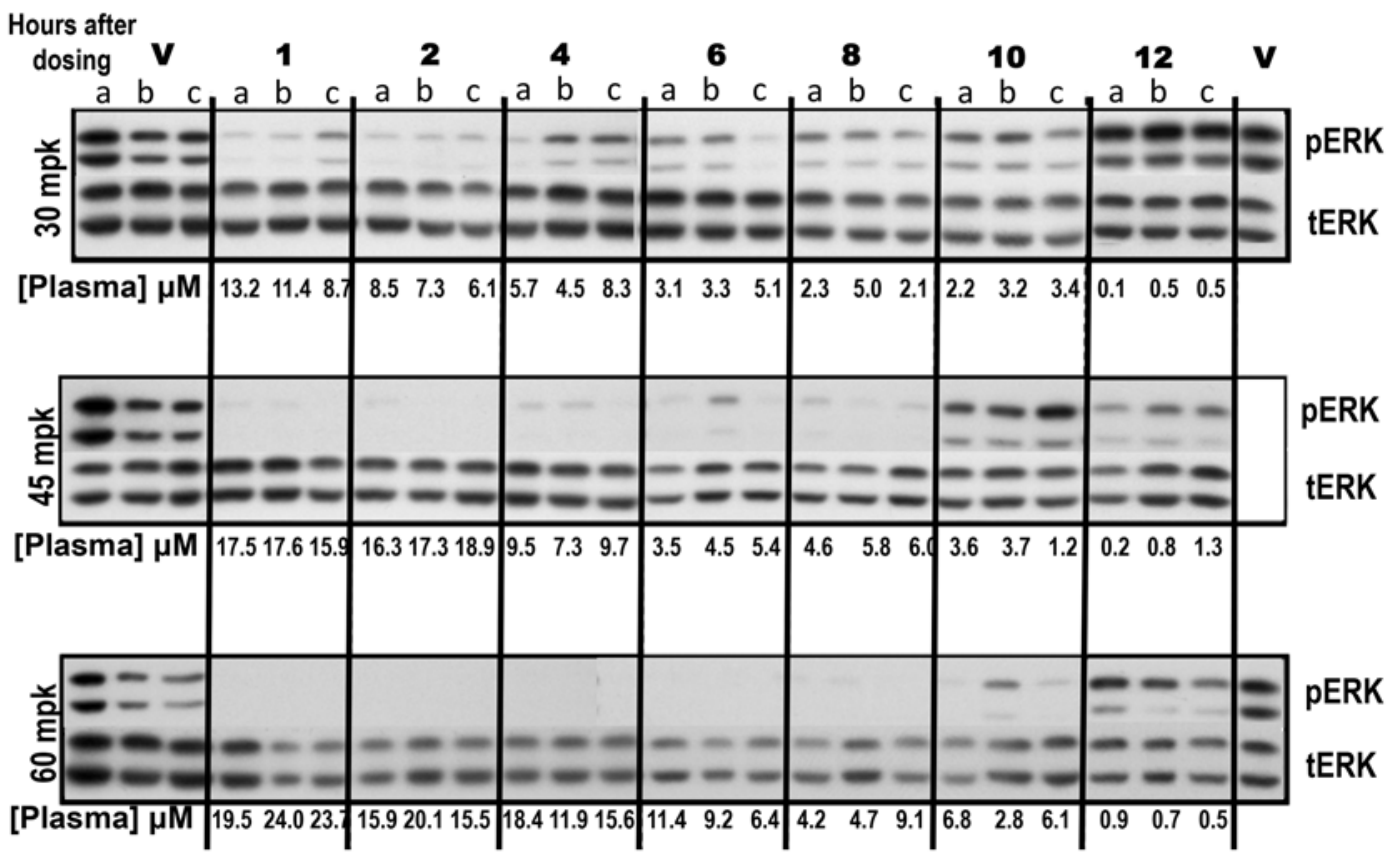

Figure 1. Structure and biochemical activity of MK-8353 in vitro and in vivo. (A) Chemical structure of MK-8353. (B) MK-8353 inhibits ERK and RSK phosphorylation. A2058 cells $\left(1 \times 10^{6}\right.$ cells per $10-\mathrm{cm}$ dish) were treated with increasing concentrations of MK-8353 (nM) for 24 hours. Whole cell lysates were subjected to immunoblot analysis using antibodies described in the Methods section. Image shows a representative immunoblot of a total of two experiments. (C and D) MK-8353 (30-60 mpk) inhibits ERK phosphorylation in Colo-205 human colon cancer tumor xenograft tissues (C) and paired normal skin tissues (D) as early as 1 hour following a single dose of MK-8353 versus drug vehicle (V). Xenograft tumors and same animal normal skin tissue were harvested (3 mice per group), and homogenates from excised tissues were subjected to immunoblot analysis using antibodies against phosphorylated/ activated ERK (pERK) and total ERK (tERK). Experiment in each tumor xenograft was performed once. See complete unedited blots in the supplemental material. 
Overall, treatment of various other human cancer xenografts with MK-8353 administered by oral gavage at $60 \mathrm{mpk}$ twice daily resulted in at least $50 \%$ tumor growth inhibition or regression in $83 \%$ of the animal models tested. In particular, treatment of Colo-205 colon cancer xenografts with MK-8353 at 30-60 mpk resulted in significant inhibition of pERK, as detected by immunoblot analysis of whole cell lysates obtained from tumor tissues as early as 1 hour following a single dose of MK-8353; however, activity of ERK1/2 rebounded after 10-12 hours (Figure 1C). Suppression of pERK in tumor tissue, as detected by Western blot analysis of corresponding tissue homogenates (Figure 1C versus Figure 1D) and by immunohistochemistry (Figure 2C versus Figure 2D; also Supplemental Figure 1), did correlate with suppression of pERK in matched skin samples from Colo-205 human colon cancer xenografts, especially as early as 2-4 hours after a single dose. In fact, modeling of the mouse preclinical data by quantitative pharmacology and pharmacometrics indicated that $>65 \%$ suppression of mean $\mathrm{pERK}$ protein expression in skin biopsies from tumor-bearing nude mice was likely to correlate with $>95 \%$ suppression of $\mathrm{pERK}$ in tumor samples and, therefore, result in meaningful clinical activity (data not shown). We therefore conclude that MK-8353 administered orally at doses ranging between 30 and $60 \mathrm{mpk}$ results in dose-proportional tumor growth inhibition or regression across various human cancer models and that twice daily dosing is required to induce sustained dose-proportional suppression of pERK in tumor tissue. Finally, detection of pERK in skin biopsies by immunohistochemistry within 2-4 hours after MK-8353 dosing can be used as a surrogate tissue pharmacodynamic biomarker of MK-8353 activity within a given window from dosing in vivo.

The pharmacokinetic parameters of MK-8353 in female nude mice were determined across various dose levels (Supplemental Table 3). Exposure levels [AUC from 0 time to 24 hours, $\left(\mathrm{AUC}_{0-24 h o u r s}\right)$ ] for a dose range between 30 and $60 \mathrm{mpk}$, the doses corresponding with both significant inhibition of $\mathrm{pERK}$ in Colo-205 human xenografts and with tumor growth inhibition or regression across various human cancer xenograft animal models, were established to be approximately 15.3-55 $\mu \mathrm{M} \cdot \mathrm{h}$ (Supplemental Figure 1). Therefore, these exposure ranges were benchmarked as the approximate exposure levels that would result in antitumor activity in cancer patients. The no-observed-adverse-effect level (NOAEL) corresponding to repeated oral twice daily administration of MK-8353 across various species/strains was quite variable but within the dose range that resulted in both biologically effective dose and tumor growth inhibition or regression in mice (Supplemental Table 4). The most conservative NOAEL from the 4-week oral rat toxicity study $(10 \mathrm{mg} / \mathrm{kg}$ ) was used to calculate the human equivalent dose, which is $1.62 \mathrm{mg} / \mathrm{kg}$. Based on a safety factor of 10 , the lowest recommended safe single dose of oral MK-8353 in healthy volunteers (average weight was considered $60 \mathrm{~kg}$ ) was $10 \mathrm{mg}$.

MK-8353 administration in healthy volunteers (P07652 study). A healthy volunteer study of MK-8353 was completed (P07652) in the Netherlands to collect toxicity, pharmacokinetic, and pharmacodynamic data. As part of this study, adult males received a single oral dose of MK-8353 in increasing (10to $400-\mathrm{mg}$ ) dose cohorts ( $10 \mathrm{mg}, n=6 ; 40 \mathrm{mg}, n=6 ; 80 \mathrm{mg}, n=12 ; 150 \mathrm{mg}, n=6 ; 300 \mathrm{mg}, n=6 ; 400$ $\mathrm{mg}, n=12$ ) in 2 alternative cohorts. In cohort 1,6 subjects received MK-8353 and 2 subjects received placebo at each dose level of $10 \mathrm{mg}, 40 \mathrm{mg}, 150 \mathrm{mg}, 400 \mathrm{mg}$, and $400 \mathrm{mg}$ plus 5 days of pantoprazole. In cohort 2, 6 subjects received an active dose and 2 subjects received placebo at each dose level of 20 $\mathrm{mg}, 80 \mathrm{mg}, 300 \mathrm{mg}, 400 \mathrm{mg}$, and $80 \mathrm{mg}$ plus food.

MK-8353 was generally safe and well tolerated across all doses, and no serious adverse events (SAEs) occurred. Adverse events (AEs) considered to be related to MK-8353 were diarrhea, pruritus, and rash. Following single-dose oral administration, peak plasma levels were attained after the median time had elapsed to reach maximum concentration ( $\left.t_{\max }\right)$ of 1.5-3 hours, which was longer than the $t_{\max }$ observed in the corresponding single-dose animal studies; the $t_{\max }$ subsequently declined biexponentially, with a terminal estimated half-life of 4.2-8.9 hours. A dose-proportional increase in peak serum concentrations $\left(C_{\max }\right)$ and the $\mathrm{AUC}_{0-}$ 24hours was noted (Supplemental Table 5). The predicted steady-state exposure of MK-8353 administered at 100 $\mathrm{mg}$ orally twice daily suggested that the $\mathrm{AUC}_{0-24 \text { hours }}$ in subjects treated with a single 100-mg dose of MK-8353 was predicted to be $16 \mu \mathrm{M} \cdot \mathrm{h}$ at steady state, which is below the NOAEL exposure level in the most sensitive species from the safety Good Laboratory Practice toxicology studies. MK-8353 administered at doses greater than or equal to $300 \mathrm{mg}$ achieved the $\mathrm{AUC}_{0-24 \mathrm{hours}}$ range of $15.5-55 \mu \mathrm{M} \cdot \mathrm{h}$; this range had been predicted to have a considerable biologic and antitumor efficacy in mice (Supplemental Table 3 and Supplemental Figure 1). This corresponds to an $\mathrm{AUC}_{0-12 \mathrm{hours}}$ of at least $12.6 \mu \mathrm{M} \cdot \mathrm{h}$, which has a substantial biological effect. However, no significant change in pERK expression was seen in hair follicles and peripheral blood mononuclear cells in any of the different dose cohorts compared with baseline (data not shown). 

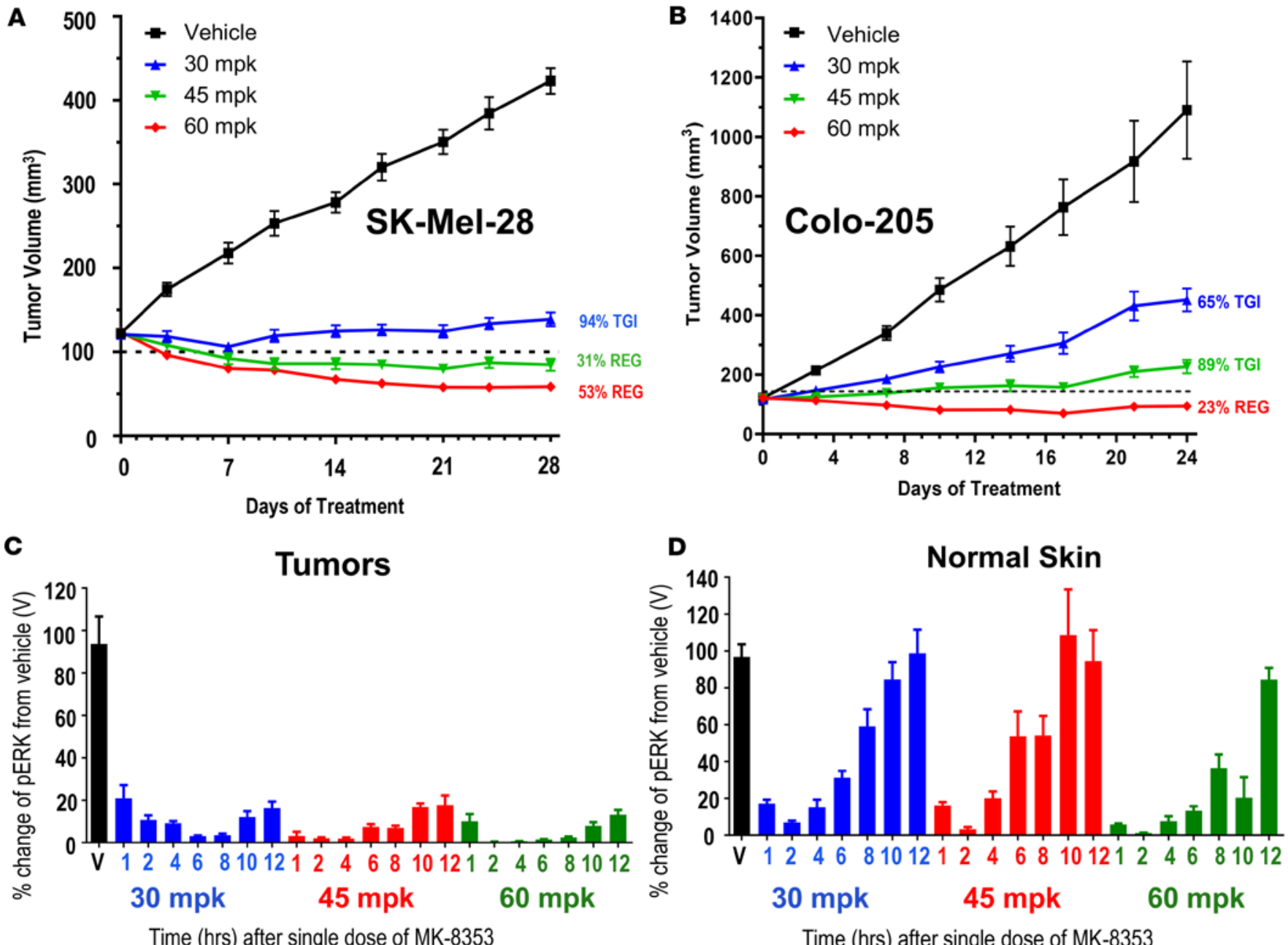

Figure 2. MK-8353 induces tumor growth inhibition or regression across various human cancer xenograft models. Female athymic nude mice (Colo-205) or SCID mice (SK-MEL-28) were injected with human Colo-205 colon cancer cells (B) and SK-MEL-28 melanoma cells (A) (both BRAF ${ }^{V 600 E}$-mutant), as described in the Methods section. (A and B). Tumor growth curves show mean tumor volumes \pm SEM (10 mice per group for each xenograft experiment). There was a statistically significant dose-dependent effect on tumor growth compared with the vehicle-treated group in both xenograft models that ranged from tumor growth inhibition (TCI) to regression (REC) and was present as early as day 3 from treatment initiation $(P<0.01$, across all time points except for the day 3 time point in the Colo-205 xenograft model, in which the 30-mpk group was significantly different, $P<0.05$; Student's $t$ test). (C and D) Expression of pERK by tissue immunohistochemistry in Colo-205 tumor xenografts (C) and same-animal normal skin tissues (D) following a single dose of MK-8353 administered by oral gavage at various doses. Representative tissue sections from corresponding tissues that were harvested at various time points ( $0-12$ hours) following a single dose of MK-8353 were stained with antibodies against pERK. See the Methods section for details and Supplemental Figure 1 for representative images of 3 animals, with tumors and normal skin imaged at each time point. Results are shown as percentage change of pERK from vehicle using the formula described in the Methods section. mpk, milligrams per kilogram; TCI, tumor growth inhibition; REG; regression; Veh, drug vehicle.

Safety and DLT in the MK-8353-001 study. Between November 2011 and February 2014, 26 patients were enrolled and 25 patients received MK-8353 (Figure 3 and Tables 1 and 2). Ten patients were treated in part 1a at doses ranging from $100 \mathrm{mg}$ to $800 \mathrm{mg}$, and 15 patients were treated in part $1 \mathrm{~b}$ at doses ranging from $200 \mathrm{mg}$ to $400 \mathrm{mg}$. Dose escalation proceeded up to the $800-\mathrm{mg}$ dose cohort, at which 2 of 6 patients experienced DLT, making us consider this dose to be beyond the MTD. Subject 1006 developed grade 3 diarrhea, hyperbilirubinemia, nausea, vomiting, and grade 2 vision changes, and subject 1010 developed grade 3 fatigue and grade 3 hyperbilirubinemia. Two of seven patients experienced DLT in the 400-mg dose cohort. Subject 2003 developed grade 2 diarrhea, which was considered to be a DLT due to several missed doses and grade 3 rash. The most frequent AEs of any grade included diarrhea (44\%), fatigue (40\%), nausea (32\%), maculopapular rash (28\%), and vomiting (28\%) (Table 3). Increased serum bilirubin was determined not be a DLT, because both drug-related and patient-related factors may have contributed. More specifically, inhibition of UGT1A1-mediated bilirubin glucuronidation was most like- 


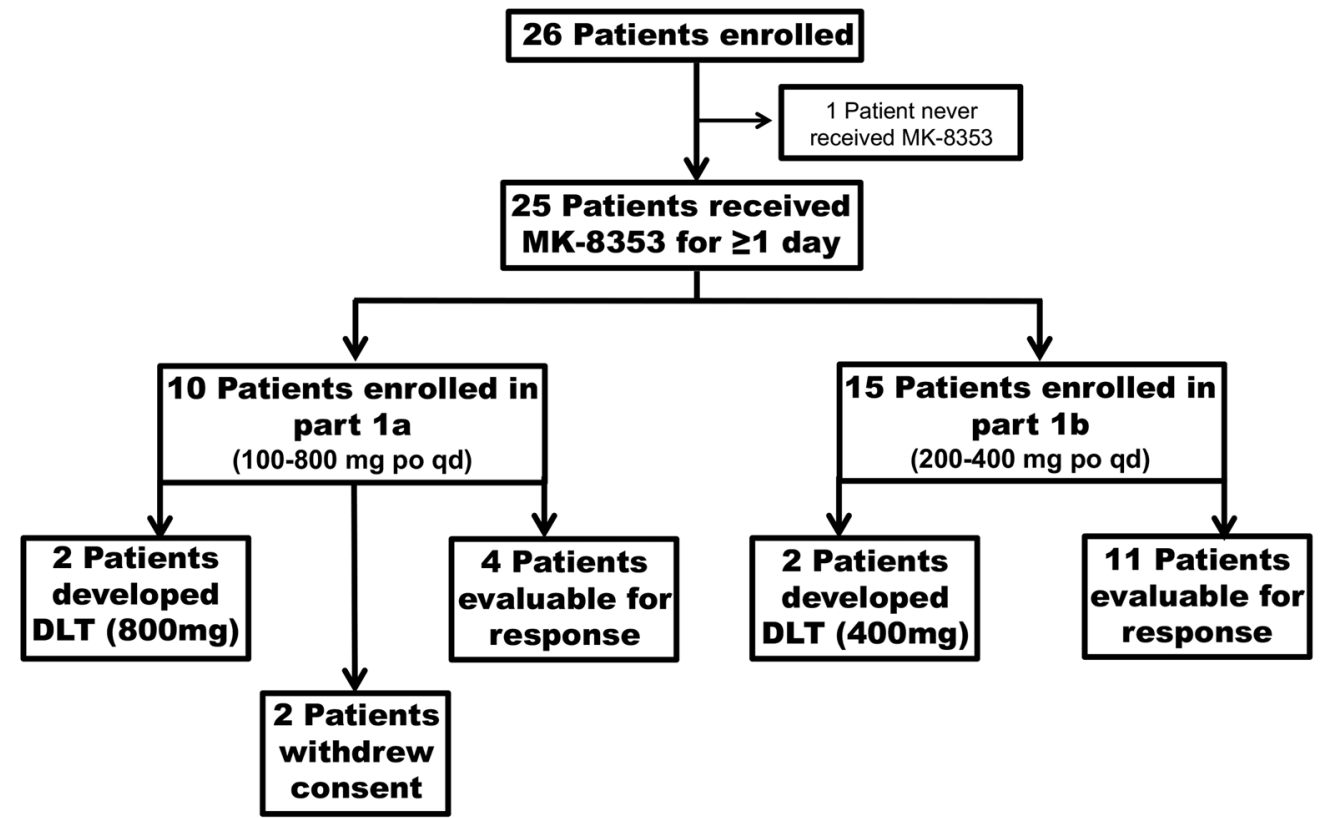

Figure 3. CONSORT diagram of the MK-8353-001 clinical trial. The flow chart shows the number of patients who were enrolled in parts $1 \mathrm{a}$ and $1 \mathrm{~b}$, received at least 1 dose of MK-8353, developed dose-limiting toxicity (DLT), and were evaluable for antitumor response by RECIST v1.1 criteria.

ly the cause of the hyperbilirubinemia observed, since some patients with elevated indirect bilirubin were found to have genetic polymorphisms in bilirubin metabolism-related genes. The most frequent grade $3 / 4$ AEs were diarrhea (16\%), isolated asymptomatic increased indirect serum bilirubin (16\%), and maculopapular rash (8\%). The 400-mg dose was identified as the preliminary MTD in part 1a. Due to early discontinuation of the study by the sponsor for strategic reasons and not due to toxicity, the RP2D was not determined. However, dosing up to $400 \mathrm{mg}$ was safe and well tolerated during part $1 \mathrm{~b}$.

Pharmacokinetic analysis of drug exposure from the MK-8353-001 study. Plasma pharmacokinetic data from 24 patients at all 6 dose levels showed that the $t_{\max }$ ranged from 2 to 5 hours, mean terminal phase half-life ranged from 5 to 14 hours, and a biexponential decline occurred after peak. Individual AUC and $C_{\max }$ values were somewhat more variable within doses compared with the healthy volunteer study, but, nonetheless, near proportional increases in their median values were observed. Notable outliers included 3 patients with unexplained rises in MK-8353 concentration from 12 to 24 hours after a single dose (subjects 2006, 2007, and 2009) and subject 1004, who exhibited substantially reduced exposure, presumably due to incomplete absorption of MK-8353 from a Whipple procedure for pancreatic cancer (Figure 4 and Supplemental Table 6). Following continuous twice daily dosing, steady-state levels were achieved by day 8 in all patients (data not shown), with an accumulation ratio of approximately 2 -fold in plasma on day 15 . Interestingly, unexplained approximately 2-fold higher exposures in cancer patients compared with healthy subject volunteers without significant differences in mean $t_{\max }$ and $t_{1 / 2}$ values were noted.

Using the $\mathrm{AUC}_{0-12 \mathrm{hour}}$ cutoff of $12.6 \mu \mathrm{M} \cdot \mathrm{h}$ as the minimum desired AUC exposure to achieve a biologically substantial effect, nearly all patients across all cohorts had sufficient dose-dependent exposure. Unfortunately, none of the 3 patients $(1005,1006$, and 1009) who were dosed with $800 \mathrm{mg}$, the dose cohort with the highest $\mathrm{AUC}_{0-12 \text { hours }}$ exposure, were on the drug long enough to assess antitumor response.

At high doses, MK-8353 (400 mg and $800 \mathrm{mg}$ orally twice daily) was found to induce unconjugated asymptomatic hyperbilirubinemia in 3 subjects (1006, 1010, and 2004). No significant differences in pharmacokinetic exposure $\left(C_{\max }, \mathrm{AUC}_{0-12 \mathrm{~h}}, t_{1 / 2}\right)$ or MK-8353 metabolism were seen in these 3 subjects compared to all other patients (Figure 4), suggesting that high pharmacokinetic exposure of the body to MK-8353 does not correlate with elevated bilirubin. Alternatively, hyperbilirubinemia may be the result of either altered metabolism by uridine $5^{\prime}$-di-glucuronosyltransferases (UGT) or altered hepatic uptake of unconjugated bilirubin. Although multiple MK-8353 glucuronides (A, B, and C) can be potentially formed via various UGT1 enzymes (UGT1A1, UGT1A3, UGT1A4), MK-8353 inhibits glucuronidation mediated by UGT1A1, a 
Table 2. Individual patient data of MK-8353-001 study subjects

\begin{tabular}{|c|c|c|c|c|c|c|c|c|}
\hline Subject no. & Cancer diagnosis & Mutation & $\begin{array}{c}\text { Prior systemic } \\
\text { treatments (BOR) }\end{array}$ & Study part & $\begin{array}{l}\text { Dose cohort } \\
\text { (mg BID) }\end{array}$ & $\begin{array}{c}\text { Evaluable for } \\
\text { efficacy }\end{array}$ & Reason for $D / C$ & $\begin{array}{c}\text { No. days on } \\
\text { MK-8353 }\end{array}$ \\
\hline 1001 & Pancreas & $K R A S^{12 V}$ & $1(\mathrm{SD})$ & $1 \mathrm{a}$ & 100 & No & $\mathrm{AE}$ & 13 \\
\hline 1002 & Colorectal & $K R A S^{W T}$ & 5 (adj/SD/unk) & $1 \mathrm{a}$ & 100 & Yes (PD) & PD & 56 \\
\hline 1004 & Pancreas & $K R A S^{12 R}$ & $1(\mathrm{PD})$ & $1 \mathrm{a}$ & 400 & Yes $(S D)^{A}$ & $\mathrm{PD}$ & 43 \\
\hline 1005 & Papill thyroid & Nontest & 2 (PD) & $1 a$ & 800 & No & $W C(A E)$ & 4 \\
\hline 1008 & Colorectal & $K R A S^{12 D}$ & $3(\mathrm{PD})$ & $1 a$ & 800 & No & $\mathrm{PD} / \mathrm{AE}$ & 4 \\
\hline 1009 & Colorectal & $K R A S^{12 V}$ & 3 (SD/PD/unk) & $1 a$ & 800 & No & $\mathrm{AE}$ & 4 \\
\hline 1010 & Colorectal & $K R A S^{125}$ & 1 (PD) & $1 a$ & 800 & No & $\mathrm{AE}$ & 5 \\
\hline 2002 & Colorectal & $K R A S^{12 D}$ & 2 (PR/PD) & $1 b$ & 400 & Yes $(P D)^{A}$ & $\mathrm{PD}$ & 14 \\
\hline 2003 & Colorectal & KRAS & 4 (adj/SD/unk) & $1 b$ & 400 & No & $W C(A E)$ & 22 \\
\hline 2008 & Melanoma & $B R A F^{600 E}$ & $1^{N}$ (adj) & $1 b$ & 300 & Yes (PR) & $\mathrm{PD}$ & 252 \\
\hline 2009 & Melanoma & $N R A S^{61 L}$ & 5 (SD/PD) & $1 \mathrm{~b}$ & 300 & No & $\mathrm{PD} / \mathrm{AE}$ & 29 \\
\hline 2010 & Melanoma & $B R A F^{600 K}$ & $3^{N}(S D / P D)$ & $1 b$ & 300 & Yes (PD) & $\mathrm{PD}$ & 55 \\
\hline 2011 & Melanoma & $B R A F^{600 K}$ & $0^{\mathrm{N}}$ & $1 \mathrm{~b}$ & 350 & Yes (PR) & $\mathrm{PD}$ & 149 \\
\hline 2012 & Melanoma & $N R A S^{61 K}$ & 2 (SD/PD) & $1 b$ & 350 & Yes (PD) & $\mathrm{PD}$ & 28 \\
\hline 2013 & Colorectal & $N R A S^{61 R}$ & 6 (SD/PD) & $1 b$ & 400 & Yes $(P D)^{A}$ & $W C(A E)$ & 28 \\
\hline 2014 & Melanoma & $B R A F^{600 E}$ & $3^{\vee}(C R / P R / P D)$ & $1 b$ & 400 & Yes (PR) & $\mathrm{PD}$ & 112 \\
\hline 2015 & Melanoma & $N R A S^{61 R}$ & 1 (PD) & $1 b$ & 350 & Yes (SD) & WC (other) & 61 \\
\hline 2016 & Melanoma & $N R A S^{61 K}$ & 2 (adj/PD) & $1 \mathrm{~b}$ & 400 & Yes (PD) ${ }^{A}$ & $\mathrm{PD} / \mathrm{AE}$ & 25 \\
\hline
\end{tabular}

NSCLC, non-small cell lung cancer; BOR, best overall therapeutic response by RECIST v1.1 criteria; adj, adjuvant therapy; SD, stable disease; unk, unknown; PD, progressive disease; PR, partial response; CR, complete response; D/C, discontinuation; WC, consent withdrawal. Prior to this study enrollment, subjects received $\left({ }^{\top}\right)$ MAPK pathway inhibitor or did not $\left({ }^{N}\right)$. ${ }^{A} A$ ntitumor response by radiographic measurements was assessed on day 28 after MK-8353 treatment.

however, his antitumor response could not be determined. Subject 2011 was 65 years old, with a history of $B R A F^{V 600 K}$-mutant melanoma without other coexisting mutations based on a 46-gene panel and no prior MAPK pathway inhibitor-based treatments. He was treated with $350 \mathrm{mg}$ MK-8353 orally twice daily and developed a PR after 2 cycles. Ultimately, he was taken off the study after he was found to have brain metastases. He developed a PR when he was subsequently treated with concurrent D+T. Subject 2014 was 58 years old with a history of $B R A F^{V 600 E}$-mutant melanoma and concurrent $C D K N 2 A^{R 58^{*}}$ nonsense mutation based on a 46-gene panel; he had previously developed complete response to single-agent vemurafenib and PR to concurrent administration of D+T. He was treated with $400 \mathrm{mg}$ MK-8353 orally twice daily following progression on $\mathrm{D}+\mathrm{T}$ and developed a PR following 2 cycles of MK-8353. However, he developed disease progression after 4 cycles and was taken off the study. Following progression on MK-8353, he developed an antitumor response to dacarbazine plus ipilimumab, no response to pembrolizumab, and, more recently, antitumor response to dabrafenib plus nivolumab.

Six patients $(2010,2005,1002,1003,2012$, and 2016) were found to have progressive disease after 2 cycles, and 3 patients $(2002,2013$, and 2004) progressed after 1 cycle. Interestingly, subject 2010, who had metastatic melanoma bearing $B R A F^{V 600 E}$ plus other mutations (e.g., GNAS $S^{S 201 H}$ and $R B 1^{E 137 D}$ mutations), and had not received any MAPK pathway inhibitor-based therapy prior to enrollment in this trial, did not respond to subsequent challenge with concurrent D+T. In contrast, the $3 B R A F^{V 600}$-mutant subjects $(2005,2008$, and 2011) that were naive to MAPK pathway inhibitors, who progressed from MK- 8353 treatment did respond to subsequent MAPK pathway inhibitor-based treatments. Of note, no other mutations other than $B R A F^{V 600}$ were identified for subjects 2008 and 2011, whereas subject 2005 was found to have $C D K 4^{R 24 L}$ missense mutation based on the TruSight Tumor 26-gene panel (Illumina). No other mutations other than the $B R A F^{V 600}$ mutation were screened in pretreatment tumors from subjects 2004 and 2007. 
Table 3. Summary of AE possibly or probably related to MIK-8353 (CTCAE v4.03) in the MK-8353-001 study

\begin{tabular}{|c|c|c|c|}
\hline \multirow[t]{2}{*}{ AE } & \multicolumn{3}{|c|}{ No. of patients having AE (\%) } \\
\hline & All grades & Grade 3 & Grade 4 \\
\hline Diarrhea & $11(44)$ & $4(16)$ & 0 \\
\hline Fatigue & $9(36)$ & $1(4)$ & 0 \\
\hline Maculopapular rash & $7(28)$ & $2(8)$ & 0 \\
\hline Nausea & $7(28)$ & $1(4)$ & 0 \\
\hline Vomiting & $6(24)$ & $1(4)$ & 0 \\
\hline Bilirubin serum, increased & $5(20)$ & $4(16)$ & 0 \\
\hline Creatinine serum, increased & $4(16)$ & 0 & 0 \\
\hline Abdominal pain & $4(16)$ & 0 & 0 \\
\hline Anemia & $3(12)$ & $1(4)$ & 0 \\
\hline Pruritus & $3(12)$ & $1(4)$ & 0 \\
\hline Decreased appetite & $3(12)$ & 0 & 0 \\
\hline Chills & $3(12)$ & 0 & 0 \\
\hline BUN serum, increased & $3(12)$ & 0 & 0 \\
\hline Dysgeusia & $3(12)$ & 0 & 0 \\
\hline Impaired gastric emptying & $3(12)$ & 0 & 0 \\
\hline Hyponatremia & $2(8)$ & 0 & 0 \\
\hline Oropharyngeal pain & $2(8)$ & 0 & 0 \\
\hline Pyrexia & $2(8)$ & 0 & 0 \\
\hline Vision blurred & $2(8)$ & 0 & 0 \\
\hline Dermatitis acneiform & $2(8)$ & 0 & 0 \\
\hline Dyspnea & $2(8)$ & 0 & 0 \\
\hline Muscular weakness & $2(8)$ & 0 & 0 \\
\hline Dehydration & $2(8)$ & 0 & 0 \\
\hline Headache & $2(8)$ & 0 & 0 \\
\hline Pain & $1(4)$ & 0 & 0 \\
\hline Increased serum AST & $2(8)$ & 0 & 0 \\
\hline Muscular weakness & $2(8)$ & 0 & 0 \\
\hline Increased serum Alk Phos & $1(4)$ & 0 & 0 \\
\hline Increase CPK & $1(4)$ & 0 & $1(4)$ \\
\hline Increased $\gamma \mathrm{GT}$ & $1(4)$ & 0 & 0 \\
\hline Dyspepsia & $1(4)$ & 0 & 0 \\
\hline Hypoalbuminemia & $1(4)$ & 0 & 0 \\
\hline Hypocalcemia & $1(4)$ & 0 & 0 \\
\hline Hypomagnesemia & $1(4)$ & 0 & 0 \\
\hline Hypermagnesemia & $1(4)$ & 0 & 0 \\
\hline Hypokalemia & $1(4)$ & 0 & 0 \\
\hline Hyponatremia & $1(4)$ & 0 & 0 \\
\hline Acute renal failure & $1(4)$ & $1(4)$ & 0 \\
\hline Amnesia & $1(4)$ & 0 & 0 \\
\hline Depressed mood & $1(4)$ & 0 & 0 \\
\hline Disorientation & $1(4)$ & 0 & 0 \\
\hline Incontinence & $1(4)$ & 0 & 0 \\
\hline Cough & $1(4)$ & 0 & 0 \\
\hline Urticaria & $1(4)$ & 0 & 0 \\
\hline
\end{tabular}

Among the 10 nonevaluable patients, subject 2009, who was diagnosed with $N R A S^{061 L}$-mutant cutaneous melanoma from the lower extremity, was noteworthy. Prior to study enrollment, she received multiple systemic treatments, including ipilimumab and pembrolizumab, without any significant antitumor response. She was enrolled into the MK-8353 trial based on in vitro drug sensitivity data from 2 melanoma cell lines that were established from her own tumors, M435-1 and M428, which showed high sensitivity to SCH772984 (Supplemental Figure 2A). Despite the initial clinical response in bulky subcutaneous tumors (Supplemental Figure 2B), she unfortunately developed symptomatic metastases to the brain, from which she subsequently died.

\section{Discussion}

We have developed an orally bioavailable, dual mechanism, direct ERK1/2 inhibitor, MK-8353, which appears to have comparable potency and selectivity with the preclinical tool compound, SCH772984 (16). Both compounds have higher potency in cellbased assays than ulixertinib (BVD-523) and CC-90003, which are currently in clinical development $(9,11)$. Comparison of the doses required for substantial biological activity (i.e., suppression of $\mathrm{pERK}$ in tissues), tumor growth inhibition or even regression, and toxicology suggest that MK-8353 administered orally twice daily may have a narrow therapeutic index, given that the drug target, ERK, is important for physiologic signaling in normal tissues (19-21) and the drug, MK-8353, binds to and inhibits both activated and nonactivated ERK.

Following an earlier study in healthy volunteers to establish preliminary toxicity, pharmacokinetic, and pharmacodynamic data (P07652), we performed a phase I study of MK-8353 in patients with advanced cancers (MK-8353-001). Pharmacokinetic analysis of the P07652 study suggested that doses between 300 and $400 \mathrm{mg}$ corresponded to drug exposure $\left(\mathrm{AUC}_{0-24 \mathrm{hour}}\right)$ that resulted in both a biologic and antitumor effect (e.g., tumor growth inhi-

bition or regression) in preclinical models. However, in the P07652 study, we did not observe any changes in $\mathrm{pERK}$ expression in either the normal skin tissue or peripheral blood mononuclear cells in the respective tissue between subjects who received either placebo or a single dose of MK-8353. We have attributed the lack of significant pharmacodynamic changes to the great variability in pERK expression among normal human subjects in different normal tissues. 

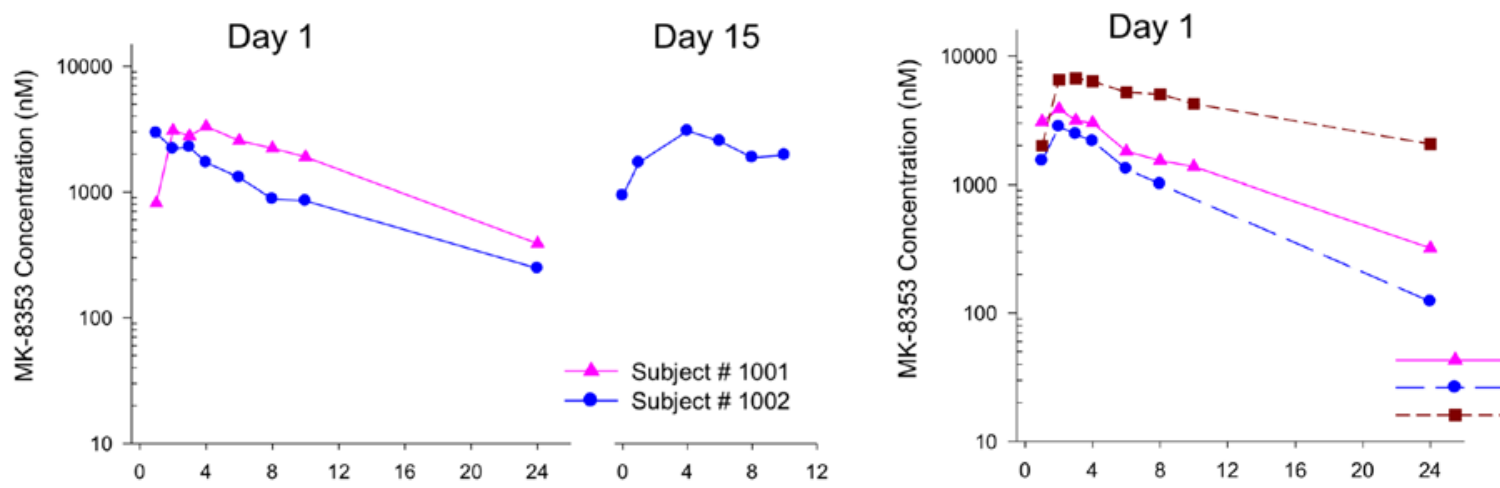

Day 15
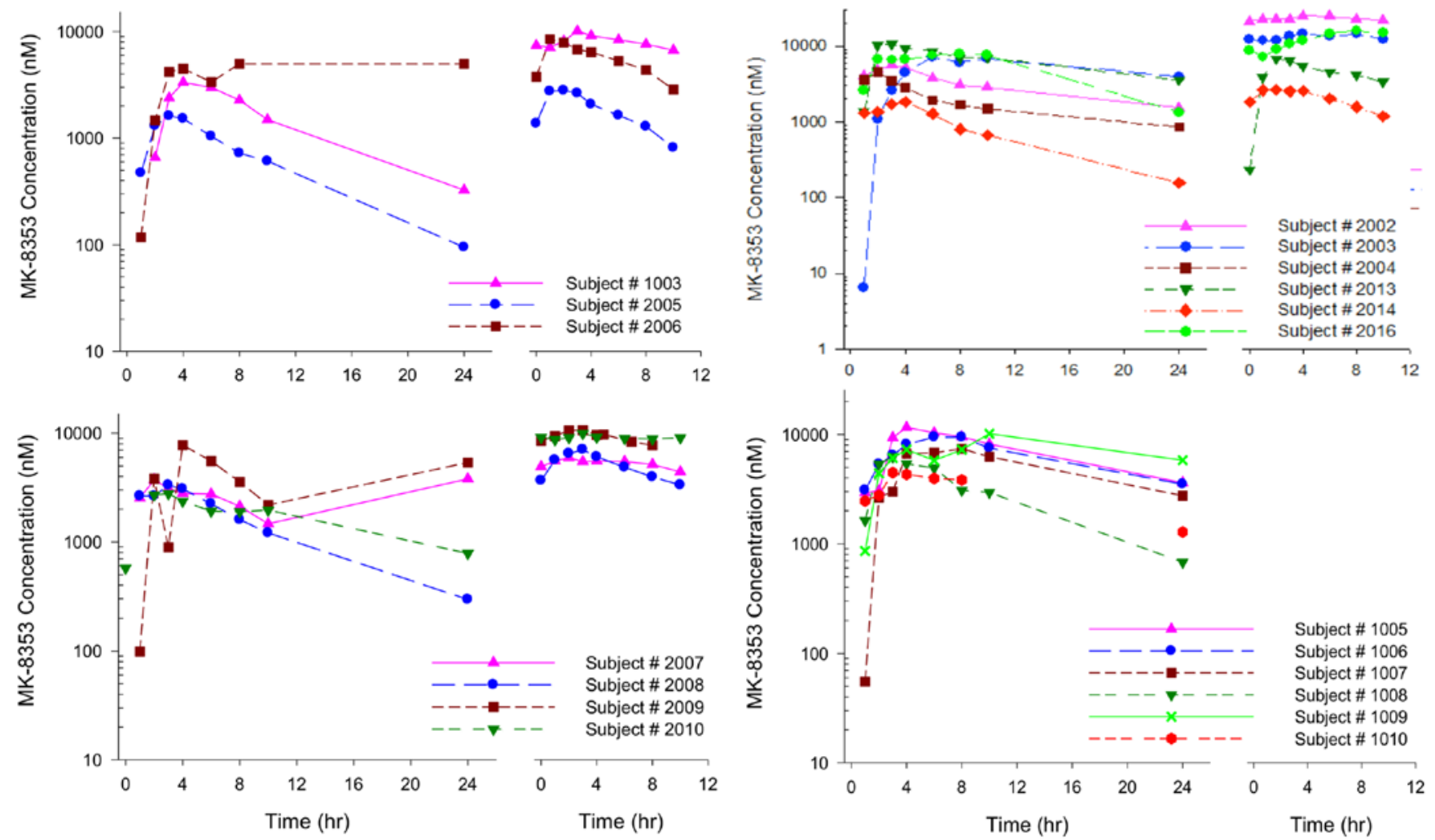

Figure 4. Mean plasma concentration-time profiles (semi-log scale) of MK-8353 in the MK-8353-001 study.

In the MK-8353-001 study, we found that MK-8353 was well tolerated at doses up to 400 mg twice daily and exhibited a side effect profile that is similar to that of MEK inhibitors (22), with diarrhea, nausea, vomiting, and rash being the most frequently seen. Although we did not establish the RP2D for MK-8353 in this study, pharmacokinetic analysis suggested adequate dose-dependent exposure across all dose cohorts according to the optimal AUC that was considered biologically effective in mouse studies. Despite the fact that 2 DLTs occurred in each of the 400-mg and 800-mg dose cohorts, no patient in the 800-mg dose cohort remained on treatment longer than 12 days; in addition, all patients in the 800-mg dose cohort stopped due to AEs (Table 2). Our inability to escalate above the 400-mg twice daily dose due to toxicity manifested as DLT or as compliance or early withdrawal from the study, which may have to do with the narrow therapeutic index of MK-8353. To compensate for the inherent high potency of this dual mechanism inhibitor that may account for patient intolerance at doses higher than $400 \mathrm{mg}$, future studies may consider continuous once daily or intermittent twice daily schedules. This alternative approach may balance higher, though more temporal suppression of pERK at the tumor tissue in favor of sparing sustained suppression of ERK signaling in normal tissues. 


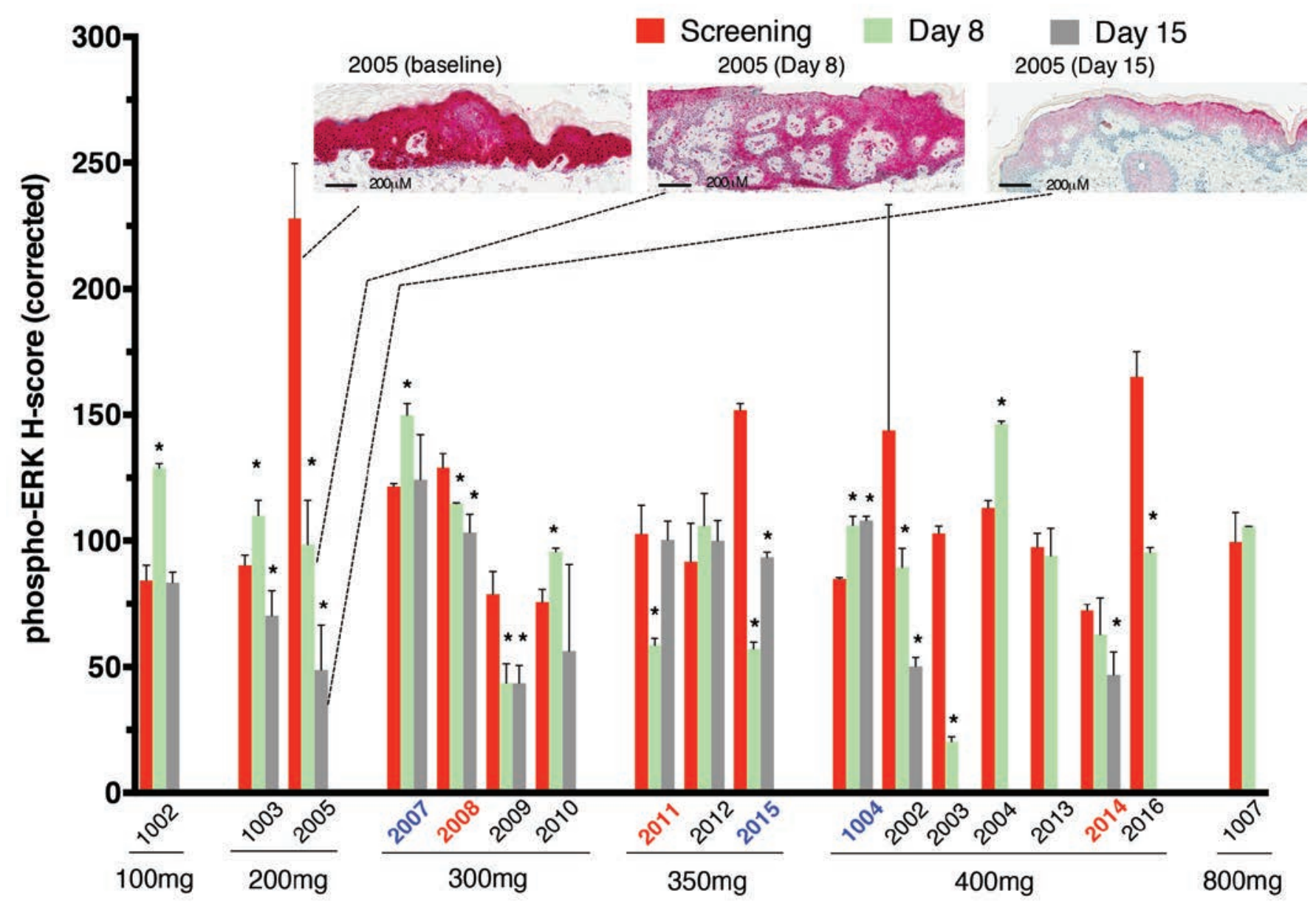

Figure 5. Effect of MK-8353 on pERK protein levels in normal epidermis in the MK-8353-001 study. Patients with the baseline (red bar) and at least 1 on-treatment biopsy (day 8, green bar; day 15, gray bar) are shown. Corrective pERK levels (H-score mean \pm SD) are shown. Asterisks show significant differences compared with baseline $\left({ }^{*} P<0.05,1\right.$-way ANOVA). Stable disease (SD) is shown in blue font; partial response (PR) is shown in red font. All other patients were nonevaluable for the efficacy endpoint. Scale bar: $200 \mu \mathrm{m}$.

Reversible unconjugated hyperbilirubinemia was seen in 3 patients in the MK-8353-001 study who received MK-8353 $\geq 400 \mathrm{mg}$ twice daily. None of the 3 subjects had history of or suspected Gilbert's syndrome, which was an exclusion criterion for our study, given the possibility of MK-8353-mediated inhibition of the UGT1A1 enzyme. Development of asymptomatic hyperbilirubinemia was not eventually determined to be a DLT and is not expected to be an issue in further clinical development of MK-8353, although close monitoring of bilirubin levels in ongoing clinical trials using MK-8353 is recommended.

Administration of $\mathrm{MK}-8353$ led to antitumor responses in 3 of 8 patients with $B R A F^{V 600}$-mutant metastatic melanoma, regardless of prior therapy with MAPK pathway inhibitor. The fact that antitumor responses were seen in doses greater or equal to $300 \mathrm{mg}$ twice daily is in line with the dose-proportional increased exposure $\left(\mathrm{AUC}_{0-12 \mathrm{hours}}\right)$ to $\mathrm{MK}-8353$. Although the number of patients with $B R A F^{V 600}$-mutant melanoma that were treated with MK-8353 as part of this study is small, the response rate was similar to what was seen in early, but much larger than the current, clinical trials testing single-agent MEK inhibitors (23). In addition, there is early evidence of temporary disease stability in patients with $N R A S^{\mathrm{Q} 61}$-mutant melanoma, although no antitumor responses were observed in any MK-8353-001 study patients bearing NRAS or KRAS-mutant cancers.

Similar to the healthy volunteer P07652 study, the advanced solid tumor MK-8353-001 study failed to show consistently significant dose-dependent suppression of pERK expression in serial normal skin biopsies in any of the doses explored. Variability in expression levels of pERK in normal skin biopsies by immunohistochemistry has been previously reported as similar or slightly lower compared with the corresponding pERK expression levels seen in melanoma biopsies (4). Normal tissues have been previously successfully 


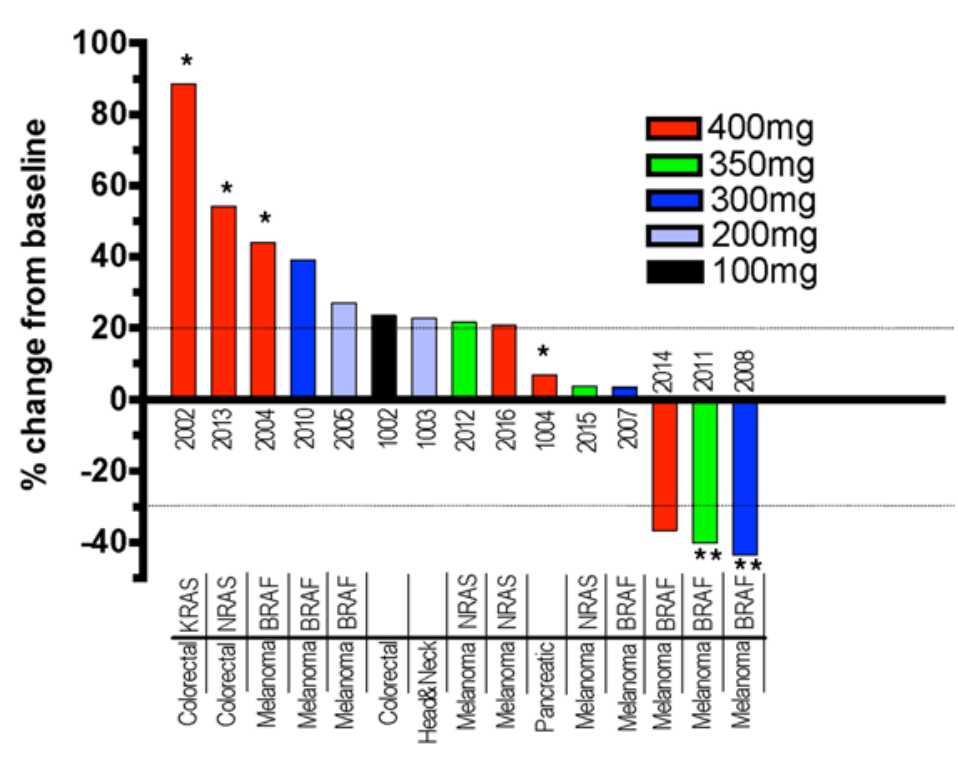

Figure 6. Investigators' assessment of objective tumor responses in evaluable patients $(n=15)$ in the MK-8353-001 study. With the exception of subjects 1004 , 2002, 2013, and 2004, whose tumor assessments were performed prior to completion of 2 cycles $(*)$, as well as subjects 2011 and 2008, whose best antitumor response occurred after 4 cycles $\left({ }^{* *}\right)$, tumor assessments were completed following completion of 2 cycles. Three patients achieved partial response $(\mathrm{PR})$.

used as pharmacodynamic markers for other targeted therapies (24), including other ERKi currently in clinical development (9). In the early clinical studies of single-agent vemurafenib in patients with $B R A F^{V 600}$-mutated metastatic melanoma, greater than $80 \%$ inhibition of cytoplasmic pERK was seen in patients with tumor regressions (25). Inhibition of $\mathrm{pERK}$ by more than $65 \%$, the presumed cut point for biologically substantial suppression of pERK based on preclinical data in tumor-bearing mice, occurred in on-treatment tissue specimens in only the 3 subjects in the 400-mg and 200-mg dose cohorts. Nevertheless, no antitumor responses were observed in those 3 patients who indeed had substantial suppression of $\mathrm{pERK}$. The comparison of the pERK immunohistochemical data between normal skin biopsies and tumor tissue in mouse studies suggests that the duration of $\mathrm{pERK}$ suppression following a single dose of MK-8353 is much shorter in normal skin compared with tumor tissue (Figure 1, C and D). This paired with the fact that the $t_{\max }$ for $\mathrm{MK}-8353$ is longer in humans compared with mice may explain why, despite our best efforts to obtain normal skin biopsies 5-7 hours after the MK- 8353 dose, we have not been able to consistently capture true pERK suppression that may have been truly achieved in normal skin biopsies before or after the actual timing of the biopsy. Since tumor tissues express more pERK than normal tissues and the duration of pERK suppression is longer in tumor tissues, analysis of pERK expression in tumor tissues is obviously expected to correlate more closely with antitumor response to MK-8353. Lack of collection of tumor tissue at baseline and at disease progression and antitumor response are all inherent weaknesses of the MK-8353-001 study. Alternatively, the lack of pERK suppression in normal skin biopsies may not be as clinically relevant, as these normal tissues may not consistently express activated-phosphorylated ERK under physiologic conditions in humans. Another important factor to consider is related with the dual mechanism of action of MK-8353; namely, any assay that solely relies on pERK expression levels does not take into account the fact that MK-8353 can also bind unphosphorylated ERK1/2, which subsequently prevents ERK1/2 phosphorylation by upstream kinases.

Molecular testing for all 3 partial responders using a 46-gene panel suggests a lack of clinically important coexisting mutations other than $B R A F^{V 600}$ that have previously shown to impair response to MAPK pathway inhibitor-based treatments (26). While coexisting mutations in clinically important genes in $B R A F^{V 600 E}$-mutant melanoma subjects 2005 and 2010 could potentially account for the lack of response to any MAPK pathway inhibitor, including MK-8353, we have no additional molecular information about coexisting mutations in the other 2 nonresponding $B R A F^{V 600}$-mutant subjects, 2004 and 2007. It is likely that additional genetic, or even epigenetic, changes may account for the lack of response to MK-8353, which were beyond the scope of the MK-8353-001 trial. For example, a detailed analysis of pERK staining over a large number of melanoma samples by immunohistochemistry showed that pERK abundance was largely heterogeneous, not only among different melanoma tumor tissues, but also within the same melanoma specimen, and did not correlate with $B R A F$ and $N R A S$ mutation status (27). Among patients with $B R A F^{V 600}$-mutant melanoma, baseline expression of pERK was also variable in melanoma biopsies (4). Similar results were seen in colorectal adenocarcinomas, although that study did not provide $R A S$ mutational status data (28). Other possibilities are 
that the ERK-dependent negative feedback on the RAF/MEK/ERK pathway is lost and/or other non-RAF/ MEK/ERK signaling pathways may alternatively contribute to oncogenic growth. In line with this, a preclinical study of the ERKi SCH772984 in KRAS-mutant pancreatic cancer suggests that both such processes may be clinically important (29). Therefore, future directions in the clinical development of ERKi might include optimizing drug formulation for better bioavailability and longer half-life, which may improve drug exposure to tumors and result in significant inhibition of pERK. However, even if such significant inhibition of pERK in tissues is achieved, combination strategies with other inhibitors of the RAF or PI3K pathway may be clinically more effective, albeit more toxic. Our preclinical data on the superiority of the combination of trametinib with SCH772984 in melanoma cell lines in vitro as well as a recent in vitro report on the synergy between trametinib and a peptide that blocks nuclear translocation of ERK1/2 (30) support possible future evaluations of concurrent MEK and ERK inhibition for several $B R A F^{V 600}$ wild-type and $B R A F^{V 600}$-mutant melanomas. Whether ERK inhibition may be more effective than MEK inhibition when each of these targets are targeted in combination with BRAF inhibitors in melanoma is currently unknown.

In conclusion, we tested the first dual-mechanism direct ERKi to our knowledge, MK-8353, in patients with advanced solid tumors, and we found that it was well tolerated at doses $\leq 400 \mathrm{mg}$ orally twice per day. $\mathrm{PK} / \mathrm{PD}$ data suggest that the dosing of MK- 8353 between $400 \mathrm{mg}$ and $800 \mathrm{mg}$ orally twice per day, or, even better, once daily, may be further explored to balance exposure, biological activity, and toxicity. The AE profile (e.g., diarrhea, fatigue, skin rash) and the antitumor activity (12\% response rate) were comparable to those seen in a larger phase I trial of ulixertinib in patients with advanced solid tumors (9). Given the promising clinical activity and the lack of SAEs, further clinical development of MK-8353 in combination with immunotherapies and for select cancer types is currently under consideration (NCT02972034). This phase I clinical study of MK-8353 provided valuable information regarding clinical activity, toxicity profile, and tolerability of MK-8353 administered twice daily; lessons about optimal pharmacodynamic endpoints; and solid tumor cancer types in which single-agent ERKi may have clinical benefit. It is likely that more ERKi will be clinically developed in the future, perhaps in combination with other targeted therapies of the MAPK or PI3K pathways for treatment of ERK-dependent cancers or even immunotherapies.

\section{Methods}

Cell lines. All cell lines used for the preclinical development of MK-8353 (Supplemental Tables 1 and 2) were purchased from ATCC. Exceptions were the UACC-62, M14, LOX, OVCAR-5, IGGROV-1, and A2780 cell lines, which were provided by the National Cancer Institute (NCI) Tumor Repository (Frederick, Maryland, USA), and the 8505C, IPC-298, SK-MEL-30, and BHT-101 cell lines, which were provided by the Leibniz-Institut DSMZ (Deutsche Sammlung von Mikroorganismen und ZellKulturen GmbH; Braunscheweig, Germany). Cell lines were maintained in culture medium as per the manufacturer's recommendations. The identities of all cell lines were confirmed using short-tandem repeat DNA analysis.

M428 and M435 are human melanoma cell lines established from baseline biopsies from MK-8353-001 study patient 2009, as previously described (31). M428 and M435 cells were cultured in RPMI 1640 with L-glutamine (Mediatech Inc.), 10\% fetal bovine serum (Omega Scientific), and 1\% penicillin, streptomycin, and fungizone (Omega Scientific) and were screened for mycoplasma contamination. Cultures were incubated in a water-saturated incubator at $37^{\circ} \mathrm{C}$ with $5 \% \mathrm{CO}_{2}$.

Drug compounds used in in vitro cell viability assays. For in vitro experiments, MK-8353 was synthesized by Merck Sharp \& Dohme Corp. SCH772984 was provided to the University of California Los Angeles (AR) through a material transfer agreement with Merck Sharp \& Dohme Corp. Trametinib was purchased from Selleck Chemicals. Stocks and solutions for all compounds were prepared in DMSO solvent to a final concentration of $10 \mathrm{mM}$. Tumor cell growth assays using MK-8353 were performed in 96-well format by plating 4,000 cells per well. On the next day cells were treated with DMSO or a 9-point IC $_{50}$ dilution (0.001-10 $\mu \mathrm{M}$ ) to a final concentration of $1 \%$ DMSO. Six replicates were assigned to each dose, and cells were treated with MK-8353 for 3 days. Cell viability was assayed using the CellTiterGlo Viability Assay luminescent kit (Promega), as per the manufacturer's instructions (17). All cell growth assays were performed 3 time for each cell line. Half-maximal effective concentration $\left(\mathrm{EC}_{50}\right)$ values were calculated using Microsoft Excel.

M428 and M435 cells were treated in duplicate with 0-10 M MK-8353 and 0-1 $\mu \mathrm{M}$ trametinib alone or in combination. A constant amount of DMSO was kept for all conditions. After incubation for 120 hours, the cell viability was determined using the CellTiterGlo Assay. Each experiment was repeated independently twice, always in duplicate. 
Animal studies. Female athymic nude mice and C57/SCID mice, both 4-6 weeks of age, were purchased from Charles River Laboratories and were maintained in a viral antibody-free barrier facility. All animals received food and water ad libitum. Regardless of the cancer cell line used, animals were inoculated subcutaneously with $5 \times 10^{6}$ cancer cells in 50\% Matrigel. Resultant tumors were grown to approximately $100 \mathrm{~mm}^{3}$ in size before initiating treatment. Mice were then randomized to treatment groups (10 mice/group) and treated by oral gavage with either MK-8353 (30-60 mpk twice daily) or 20\% HPBCD vehicle twice daily. Tumor length $(L)$, width $(W)$, and height $(H)$ were measured using a caliper twice weekly on each mouse. Animal body weight was measured twice weekly for the duration of treatment.

Antibodies and protein detection. For immunoblot analysis MK-8353-treated cells were lysed in radioimmunoprecipitation assay buffer with protease inhibitor cocktail (Roche Diagnostics $\mathrm{GmbH}$ ) and cleared by centrifugation. Protein concentration was determined using bicinchoninic acid reagent (Pierce Chemical). Protein samples were separated by $10 \%$ SDS-PAGE and transferred onto a nitrocellulose membrane. Blots were incubated with primary antibodies against phosphorylated ERK1/2 T185/Y187 (rabbit polyclonal, BioSource/Thermo Fisher Scientific, catalog no. 44-680G, dilution 1:1,000), ERK1/2 (rabbit polyclonal, BioSource/Thermo Fisher Scientific, catalog no. 44-654G, dilution 1:1,000), phosphorylated p90 RSK T359/S363 (rabbit monoclonal, clone E238, Cell Signaling Technology, catalog no. 9344, dilution 1:500), total RSK (mouse monoclonal, clone 78/RSK, BD Transduction Laboratories, catalog no. 610226, dilution 1:5,000), and cleaved poly (ADP-ribose) polymerase p85 fragment (rabbit polyclonal, Promega, catalog no. G7341, dilution 1:2,000). Following incubation with primary and secondary antibodies, band intensity was detected by chemiluminescence using enhanced chemiluminescence detection reagents (Amersham). Densitometric quantification of each band was determined using Quantity One software (PDI Inc.) and was normalized in comparison with the relevant expression of $\beta$-actin in the reprobed blot.

For immunohistochemical detection of phospho-ERK, 5 - $\mu \mathrm{M}$-thick formalin-fixed paraffin-embedded tissue sections (tumor tissue and normal skin) were deparaffinized and rehydrated by serial passages through ClearRite (Thermo Fisher Scientific) (5 minutes, 3 times), 100\% Flex (5 minutes, twice), 95\% Flex (Thermo Fisher Scientific) (5 minutes, once), $80 \%$ Flex ( 5 minutes, once), $50 \%$ Flex (3 minutes, once), and $\mathrm{dH}_{2} 0$ ( 5 minutes) solutions. Antigen retrieval was performed in decloaking chamber at $98^{\circ} \mathrm{C}$ for 35 minutes using the DAKO Target Retrieval Solution (Dako/Agilent Pathology Solutions, catalog no. S1700). Tissue slides were then cooled for 5 minutes, rinsed with $\mathrm{dH}_{2} \mathrm{O}$ for 20 minutes, and subsequently incubated for 5 minutes with $0.03 \% \mathrm{H}_{2} \mathrm{O}_{2}$. Tissue slides were then incubated with $200 \mu \mathrm{l}$ diluted (1:100) primary antibody against phospho-ERK (T202/Y204) (rabbit monoclonal, clone D13.14.4E, Cell Signaling, catalog no. 4370) in DAKO antibody diluent (Dako/ Agilent Pathology Solutions, catalog no. S0809) for 30 minutes followed by incubation with peroxidase-labeled polymer goat anti-rabbit for 30 minutes. HRP-labeled polymer anti-rabbit (DAKO EnVIsion+ System, Dako/ Agilent Pathology Solutions, catalog no. K4003) solution (200 $\mu 1$ per tissue slide) was then added for 5 minutes. Slides were then rinsed and counterstained with hematoxylin QS (Vector Labs, catalog no. H3404) for 1 minute. Tissue slides were finally rinsed with $\mathrm{dH}_{2} \mathrm{O}$, dehydrated by serial passages through $70 \%$ Flex (20 seconds, once), 95\% Flex (20 seconds, once), 100\% Flex (20 seconds, twice), and Clear-Rite (1 minute, twice) and coverslipped. Five digital images were obtained from each immunohistochemically stained tissue section (tumor tissue and normal skin; 3 mice per each time point) using Zeiss Axioskop light microscope equipment. Signal was quantified using AxioVision software (Carl Zeiss Microscopy LLC).

Patients (MK-8353-001 study). Patients were recruited either via self-referral, referral by community medical oncologists, or as established patients across 9 academic institutions on 3 continents. Eligibility required a histologic diagnosis of advanced solid tumors, adequate organ function (hemoglobin $\geq 9.0 \mathrm{gm} / 1$, absolute neutrophil count $\geq 1,500 / \mu 1$, platelet count $\geq 100,000 / 1$, serum cystatin $C$ level $\leq 1.5 \times$ upper limits of normal [ULN], aspartate aminotransferase and alanine aminotransferase $\leq 5 \times$ ULN if liver metastases were present or $\leq 3 \times$ ULN if absent, and bilirubin $2 \times$ ULN, unless Gilbert's syndrome was suspected), ECOG performance status of 0 or 1 , and the ability to swallow and absorb oral medications. Subjects with unstable or progressing brain metastases, gastrointestinal disorder that may alter drug absorption, QTcF interval $>450 \mathrm{~ms}$, and history of retinal vein occlusion, central serous retinopathy, or glaucoma or female subjects who were breastfeeding, pregnant, or intending to become pregnant were excluded. Investigators from each study site were required to provide subject data to the study sponsor, Merck Sharp \& Dohme Corp., according to Sponsor's instructions and compliant with Good Clinical Practice guidelines.

Study design (MK-8353-001 study). The trial (NCT01358331) was conducted in 2 parts. Part 1a enrolled subjects with any incurable advanced solid tumor. In part 1a, the MK-8353 dose was increased, and 
MTD range was preliminarily determined based on safety, tolerability, and pharmacokinetic/pharmacodynamics evaluations. Part $1 \mathrm{~b}$ enrolled patients with melanoma and colon cancer bearing oncogenic hotspot RAS and/or BRAF mutations. Patients in part $1 \mathrm{~b}$ must have had at least one measurable lesion as defined by RECIST v1.1 criteria and should have not received greater than 3 prior systemic treatments, excluding MAPK pathway inhibitor. Assessment of $B R A F^{V 600}$ or $R A S$ mutation status was performed in a Clinical Laboratory Improvement Amendments-certified laboratory or by centralized testing. The previously defined MTD range from part 1a would be further refined and defined in part $1 \mathrm{~b}$ for subsequent phase II studies. Patients without any prior systemic treatment for their cancer were allowed to participate at any dose cohort.

Study drug administration (07652 and MK-8353-001 studies). MK-8353 was supplied in capsules by Merck Sharp \& Dohme Corp., a subsidiary of Merck \& Co. Inc., and was administered orally on an empty stomach 1 hour before or approximately 2 hours after a meal. In the 07652 study, MK-8353 was administered as a single dose to healthy adult males in the outpatient clinic, whereas in the MK-8353001 study, MK-8353 was administered twice daily at home or in the outpatient clinic continuously in consecutive 28-day cycles until disease progression or unacceptable toxicity. In part 1a of the MK-8353001 study, dose escalation followed an accelerated titration design. As long as no grade 2 toxicities occurred, the next dose level would be double that of the previous dose level. If a single $\geq$ grade 2 toxicity (non-dose-limiting) occurred in any given dose cohort, the dose level would expand to 3 subjects. If no additional $\geq$ grade 2 toxicity (non-dose-limiting) occurred, dose escalation would continue by doubling of the previous dose level. If one or more additional $\geq$ grade 2 toxicity(-ies) (in 2 or 3 of the 3 patients) occurred, dose escalation would continue to increase the dose by approximately $67 \%, 50 \%, 33 \%$, and $25 \%$, respectively. In part $1 \mathrm{~b}$ of the MK-8353-001 study, dose level started at the preliminary MTD with potential for dose escalation, depending on the number of subjects evaluable for analysis for DLT. DLT was counted toward RPTD decision in dose escalation.

Safety and response assessment (MK-8353-001 study). The prespecified safety endpoint was the DLT rate occurring during the first cycle. For determination of AE severity, the NCI's Common Terminology Criteria for Adverse Event (NCI-CTCAE) v4.03 was used. The preliminary MTD was defined as the highest dose level for which DLT is $<33 \%$. DLT was defined as any $\geq$ grade 3 hematologic toxicity lasting $\geq 1$ week; any $\geq$ grade 3 nonhematologic toxicity not clearly attributable to other causes; grade 3 nausea, vomiting, and anorexia, if maximal supportive care had been delivered; any grade 4 nonhematological laboratory value not related to underlying disease; $>20 \%$ missed doses due to toxicity in the first cycle. Response rate was a secondary endpoint for this trial and was defined by RECIST v1.1. Imaging was performed every other cycle, unless the investigator felt clinical need for earlier assessments.

Pharmacokinetic assessments (tumor growth xenograft, 07652, and MK-8353-001 studies). For pharmacokinetic analysis, plasma was collected on cycle 1 , day 1 (0, 1, 3, 4, 6, 8, 10, and 24 hours; 07652 and MK-8353-001 studies), and day 15 (immediately before the first dose on that day and 1, 2, 3, 4, 6, 8, and 10 hours after the first dose; MK-8353-001 study) of MK-8353 treatment. Plasma MK-8353 concentrations were used to estimate the following pharmacokinetic parameters of MK-8353 treatment: maximum observed plasma concentration $\left(C_{\max }\right)$, time-to-maximum observed plasma concentration $\left(t_{\max }\right)$, area-under-the-plasma concentration-time curve, apparent total body clearance $(\mathrm{CL} / \mathrm{F})$, terminal phase half-life $\left(t_{1 / 2}\right)$, accumulation ratio, and apparent volume of distribution $(\mathrm{Vd} / \mathrm{F})$.

The analytical method for the analysis of MK-8353 was based on an automated 96-well format liquid-liquid extraction of drug from human plasma. Briefly, drug and internal standard were extracted from plasma with methyl tert-butyl ether. Samples were mixed-rotated and centrifuged in a 96 -well plate. The organic supernatant layer was removed, evaporated under nitrogen, and reconstituted in 50:50 acetonitrile/ water. The plate was sealed, and $2-10 \mu 1$ of the reconstituted sample was injected onto a high-performance liquid chromatography tandem mass spectrometry system for analysis. Drug and internal standard were chromatographed using reverse-phase liquid chromatography and detected with tandem mass spectrometry. The multiple reaction monitoring transitions were monitored with mass-to-charge ratio $(\mathrm{m} / \mathrm{z}) 692.2 \rightarrow$ 424.1 for MK-8353. The lower limit of quantitation for this method was $2 \mathrm{ng} / \mathrm{ml}$.

Pharmacodynamic assessments in clinical studies (07652 and MK-8353-001). The degree of pERK suppression, as detected by immunohistochemistry in normal 4-mm skin punch biopsies, was used as a surrogate biomarker of ERK activation at the following time points: at baseline, on cycle 1, and on days 8 and 15. Immunohistochemistry was performed on a Ventana Discovery XT immunostainer 
(Ventana Medical Systems Inc.) using the DISCOVERY ChromoMap RED kit Alkaline Phosphatase (AP) staining kit (Ventana). Slides were deparaffinized, underwent heat-induced epitope retrieval using the RiboCC buffer (Ventana), and were incubated in 7\% goat serum (MilliporeSigma) for 20 minutes to inhibit nonspecific staining. Slides were subsequently incubated with rabbit monoclonal antibody against phospho-ERK (clone D13.14.4E, Cell Signaling; 1:600 dilution) or rabbit monoclonal antibody IgG isotype control (DA1E, Cell Signaling) for 60 minutes. Antibody detection was performed using the DISCOVERY UltraMap anti-Rabbit AP detection system (Ventana). Tissues were counterstained with Hematoxylin II (Ventana) and developed using bluing reagent (Ventana). Stained slides were transferred to a Leica Multistainer/Coverslipper (ST5020/CV5030, Leica Microsystems Inc.), dehydrated in graded ethanol and xylene, and coverslipped.

Immunohistochemically stained slides for pERK1/2 from both xenograft and clinical studies were scanned into Aperio ScanScope XT. Digital images were analyzed using Aperio ImageScope software (Leica Microsystems Inc.). Analysis algorithm was developed using a panel of human skin tissues. Staining was evaluated using computer imaging analysis (color deconvolution algorithm) in which electronic gates were manually set to select for epidermis and exclude dermal and subdermal regions and structures. From each skin biopsy, two slides were used to stain $\mathrm{pERK}$ and one slide was used to stain for IgG negative control. A single lot of the pERK antibody was used for staining.

Statistics. Descriptive analysis of preclinical data was performed using GraphPad Prism 5.0 (GraphPad Software Inc.). $\mathrm{EC}_{50}$ were calculated using Microsoft Excel. Tumor volumes in animal experiments were calculated using the formula $(L \times W \times H) / 2$. Tumor volume data are expressed as mean \pm SEM. Statistically significant differences in tumor growth curves and immunohistochemical expression of $\mathrm{pERK}$ among the different treatment groups in xenograft studies were determined by the Student's $t$ test. An unadjusted $P$ value of 0.05 was considered as statistically significant.

Descriptive analysis of clinical data was performed using the Statistical Analysis System (SAS) software suite release 9.1 (SAS Institute). Analysis of pharmacokinetic data was performed using Phoenix WinNonLin (Certara). Graphs were developed using Prism 6.0 (GraphPad Software Inc.). Subjects who received $\geq 1$ dose of MK-8353 and had $\geq 1$ efficacy assessment after baseline were included in the efficacy endpoint analysis, whereas subjects who received $\geq 1$ dose of MK- 8353 and completed cycle 1 or discontinued due to toxicity were evaluable for DLT. The DLT rates along with the $80 \%$ Bayesian credible intervals for the DLT rate in cycle 1 were provided. Plasma MK-8353 concentrations and pharmacokinetic parameters were listed and summarized by dose using descriptive statistics. The pharmacokinetic parameters AUC and $C_{\max }$ were log-transformed and analyzed based on a linear-mixed effects model containing a fixed effect for treatment and a random effect for subject.

For pharmacodynamic analysis of pERK inhibition by immunohistochemistry in normal human skin biopsies, the color deconvolution algorithm was used to assess staining on a "per pixel" basis and is reported as a score calculated in a manner similar to an H-score:

Score $=[(\% 1+$ pixels $) \times 1]+[(\% 2+$ pixels $) \times 2]+[(\% 3+$ pixels $) \times 3]$

Corrected score was defined as follows: (pERK score) - (IgG-negative control score). Percentage inhibition was then calculated using the corrected scores.

Study approval. The MK-8353-001 study protocol was approved by the IRBs of the University of North Carolina at Chapel Hill, Massachusetts General Hospital Cancer Center, The University of Texas MD Anderson Cancer Center, the Translational Genomics Research Institute, the Virginia G. Piper Cancer Center, the Roswell Park Cancer Institute, The University of Auckland and Auckland City Hospital, the Chaim Sheba Medical Center, the University of Pittsburgh School of Medicine, Merck Sharp \& Dohme Corp., and the Jonsson Comprehensive Cancer Center at UCLA. M428 and M435 human melanoma cell lines were established under the University of California Los Angeles IRB (02-08-067)). Written informed consent was received from participants prior to inclusion in the study. Written informed consent was also provided for patient pictures appearing in the manuscript. Animal procedures were performed according to the NIH Guide For The Care And Use of Laboratory Animals (National Academies Press, 2011).

\section{Author contributions}

TSR, DZ, NRM, AAS, EHR, JS, BJL, PD, DC, PK, WRB, YD, AC, and GWS were involved in preclinical development of MK-8353. JR, AAS, PC, and EHR were involved in the healthy volunteer study plan and conduct. JR, PC, AAS, EHR, and KTF were involved in the MK-8353-001 study planning and conduct. 
SJM, RJS, WJH, RKR, AAA, PCF, RSF, HAT, AR, and KTF were involved in patient enrolling, care, and assessment, as well as reporting of antitumor response and toxicity to MK-8353. TSR, DZ, NRM, AAS, BHM, LR, and AR were involved in the correlative analyses. SJM, RJS, WJH, JR, NRM, AAS, BJL, AR, and KTF were involved in manuscript writing, editing, and submission. SJM, RJS, BJL, AAS, EHR, AR, and KTF are responsible for the overall content.

\section{Acknowledgments}

We thank Channing Der, Kennan Distinguished Professor, University of North Carolina at Chapel Hill, for his useful comments on the manuscript and Colleen MacDonagh for providing editorial assistance. We also thank Lijuan Liu and Alexander Stoeck (Merck \& Co. Inc.) for their assistance with data collection. Cell viability studies for the M428 and M435 patient-derived melanoma cell lines were funded by NIH grants P01 CA168585 and R35 CA197633 (to AR). We thank the healthy volunteers and the patients who participated in this study and made possible the clinical evaluation of MK-8353. This study was supported financially by Merck Sharp \& Dohme Corp.

Address correspondence to: Keith T. Flaherty, Massachusetts General Hospital, 55 Fruit Street, Yawkey 9E, Boston, Massachusetts 02114-2696, USA. Phone: 617.726.1941; Email: kflaherty@partners.org. Or to: Stergios J. Moschos, University of North Carolina at Chapel Hill, 170 Manning Drive, CB\# 7305, Chapel Hill, North Carolina 27599, USA. Phone: 919.843.7713; Email: moschos@med.unc.edu.

RKR's present address is: Mayo Clinic Hospital, Phoenix, Arizona, USA.

AAA's present address is: Mayo Clinic, Rochester, Minnesota, USA.

HAT's present address is: The University of Texas MD Anderson Cancer Center, Houston, Texas, USA.

TSR's present address is: GlaxoSmithKline, Philadelphia, Pennsylvania, USA.

AAS's present address is: TheraMet Biosciences LLC, Lewes, Delaware, USA.

PC's present address is: Gilead Sciences, San Mateo, California, USA.

PK's present address is: Belfer Center for Applied Cancer Science, Dana-Farber Cancer Institute, Boston, Massachusetts, USA.

WRB's present address is: Synergy Partners R\&D Solutions, New York, New York, USA.

AC's present address is: Bantam Pharmaceutical LLC, New York, New York, USA.

GWS's present address is: Shire, Lexington, Massachusetts, USA.

BHM's present address is: Merck \& Co. Inc., Kenilworth, New Jersey, USA.

1. Yoon S, Seger R. The extracellular signal-regulated kinase: multiple substrates regulate diverse cellular functions. Growth Factors. 2006;24(1):21-44.

2. McKay MM, Morrison DK. Integrating signals from RTKs to ERK/MAPK. Oncogene. 2007;26(22):3113-3121.

3. Kandoth C, et al. Mutational landscape and significance across 12 major cancer types. Nature. 2013;502(7471):333-339.

4. Trunzer K, et al. Pharmacodynamic effects and mechanisms of resistance to vemurafenib in patients with metastatic melanoma. J Clin Oncol. 2013;31(14):1767-1774.

5. Ahronian LG, et al. Clinical acquired resistance to raf inhibitor combinations in BRAF-mutant colorectal cancer through MAPK pathway alterations. Cancer Discov. 2015;5(4):358-367.

6. Carlino MS, et al. Differential activity of MEK and ERK inhibitors in BRAF inhibitor resistant melanoma. Mol Oncol. 2014;8(3):544-554.

7. Shima F, Matsumoto S, Yoshikawa Y, Kawamura T, Isa M, Kataoka T. Current status of the development of Ras inhibitors. $J$ Biochem. 2015;158(2):91-99.

8. Mita MM, et al. A phase Ia study of CC-90003, a selective extracellular signal-regulated kinase (ERK) inhibitor, in patients with relapsed or refractory BRAF or RAS-mutant tumors. J Clin Oncol. 2017; 35(15_suppl):2577.

9. Sullivan RJ, et al. First-in-class oral ERK1/2 inhibitor ulixertinib (BVD-523) in patients with MAPK mutant advanced solid tumors: results of a phase I dose-escalation and expansion study. Cancer Discov. 2018;8(2):184-195.

10. Herrero A, et al. Small molecule inhibition of erk dimerization prevents tumorigenesis by RAS-ERK pathway oncogenes. Cancer Cell. 2015;28(2):170-182.

11. Germann UA, et al. Targeting the MAPK signaling pathway in cancer: promising preclinical activity with the novel selective ERK1/2 inhibitor BVD-523 (ulixertinib). Mol Cancer Ther. 2017;16(11):2351-2363.

12. Blake JF, et al. Discovery of (S)-1-(1-(4-Chloro-3-fluorophenyl)-2-hydroxyethyl)-4-(2-((1-methyl-1H-pyrazol-5-yl)amino)pyrimidin-4-yl)pyridin-2(1H)-one (GDC-0994), an Extracellular Signal-Regulated Kinase 1/2 (ERK1/2) Inhibitor in Early Clinical Development. J Med Chem. 2016;59(12):5650-5660.

13. Miermont AM, Pflicke H, Guan H, Chinnasamy H, Thomas C, Rudloff U. Abstract 5538: Direct inhibition of ERK1/2 by VTX-11e leads to increased induction of apoptosis in a subset of pancreatic cancer cell lines as compared to MEK1/2 inhibi- 
tion by selumetinib (AZD6244). Cancer Res. 2013;73(8 Supplement):5538.

14. Samatar AA, Poulikakos PI. Targeting RAS-ERK signalling in cancer: promises and challenges. Nat Rev Drug Discov. 2014;13(12):928-942.

15. Chaikuad A, et al. A unique inhibitor binding site in ERK1/2 is associated with slow binding kinetics. Nat Chem Biol. 2014;10(10):853-860.

16. Morris EJ, et al. Discovery of a novel ERK inhibitor with activity in models of acquired resistance to BRAF and MEK inhibitors. Cancer Discov. 2013;3(7):742-750.

17. Wong DJ, et al. Antitumor activity of the ERK inhibitor SCH772984 [corrected] against BRAF mutant, NRAS mutant and wild-type melanoma. Mol Cancer. 2014;13:194.

18. Zhang D, Chando TJ, Everett DW, Patten CJ, Dehal SS, Humphreys WG. In vitro inhibition of UDP glucuronosyltransferases by atazanavir and other HIV protease inhibitors and the relationship of this property to in vivo bilirubin glucuronidation. Drug Metab Dispos. 2005;33(11):1729-1739.

19. Masaki T, Stambe C, Hill PA, Dowling J, Atkins RC, Nikolic-Paterson DJ. Activation of the extracellular-signal regulated protein kinase pathway in human glomerulopathies. J Am Soc Nephrol. 2004;15(7):1835-1843.

20. de Melo M, Gerbase MW, Curran J, Pache JC. Phosphorylated extracellular signal-regulated kinases are significantly increased in malignant mesothelioma. J Histochem Cytochem. 2006;54(8):855-861.

21. Huttlin EL, et al. A tissue-specific atlas of mouse protein phosphorylation and expression. Cell. 2010;143(7):1174-1189.

22. Flaherty KT, et al. Improved survival with MEK inhibition in BRAF-mutated melanoma. N Engl J Med. 2012;367(2):107-114

23. Falchook GS, et al. Activity of the oral MEK inhibitor trametinib in patients with advanced melanoma: a phase 1 dose-escalation trial. Lancet Oncol. 2012;13(8):782-789.

24. Agulnik M, et al. Predictive and pharmacodynamic biomarker studies in tumor and skin tissue samples of patients with recurrent or metastatic squamous cell carcinoma of the head and neck treated with erlotinib. J Clin Oncol. 2007;25(16):2184-2190.

25. Bollag G, et al. Clinical efficacy of a RAF inhibitor needs broad target blockade in BRAF-mutant melanoma. Nature. 2010;467(7315):596-599.

26. Nathanson KL, et al. Tumor genetic analyses of patients with metastatic melanoma treated with the BRAF inhibitor dabrafenib (GSK2118436). Clin Cancer Res. 2013;19(17):4868-4878.

27. Houben R, Vetter-Kauczok CS, Ortmann S, Rapp UR, Broecker EB, Becker JC. Phospho-ERK staining is a poor indicator of the mutational status of BRAF and NRAS in human melanoma. J Invest Dermatol. 2008;128(8):2003-2012.

28. Tai CJ, et al. High nuclear expression of phosphorylated extracellular signal-regulated kinase in tumor cells in colorectal glands is associated with poor outcome in colorectal cancer. Ann Diagn Pathol. 2013;17(2):165-171.

29. Hayes TK, et al. Long-term ERK inhibition in KRAS-mutant pancreatic cancer is associated with MYC degradation and senescence-like growth suppression. Cancer Cell. 2016;29(1):75-89.

30. Arafeh R, et al. Combined inhibition of MEK and nuclear ERK translocation has synergistic antitumor activity in melanoma cells. Sci Rep. 2017;7(1):16345

31. Søndergaard JN, et al. Differential sensitivity of melanoma cell lines with BRAFV600E mutation to the specific Raf inhibitor PLX4032. J Transl Med. 2010;8:39. 